\title{
Correlation between modified CT severity index and retroperitoneal extension using the interfascial planes in the grading of clinically suspected acute severe pancreatitis
}

\author{
Nesreen Mohey and Tamir A. Hassan * (D)
}

\begin{abstract}
Background: This prospective study was conducted between January 2015 and June 2018 and included 50 patients (mean age of $52.12 \pm 2.0$ years), all presented with acute pancreatitis and admitted to the ICU 1-3 days after the onset. All patients underwent contrast-enhanced $\mathrm{CT}$, and images are evaluated by 2 independent radiologists for the modified CTSI parameters and retroperitoneal extension parameters to detect the severity of pancreatitis. The aim of this study is to compare the results of the modified CT severity index and retroperitoneal interfascial extension $C T$ grading system in the grading of the severity of acute pancreatitis and compare both grading systems to the clinical course of the disease and its complications.
\end{abstract}

Results: Both grading systems show a statistical significant correlation in terms of the length of hospital stay, the need for interventions, and the development of organ failure.

Conclusion: In conclusion, a good correlation was observed between the modified CTSI and retroperitoneal extension grading systems in grading the severity of acute pancreatitis. We think that we can apply Ishikawa et al. grading system as a prognostic indicator if there is a contraindication to contrast administration.

Keywords: Modified CT severity index, Acute pancreatitis, Retroperitoneal interfascial planes, Severity of acute pancreatitis, Grading of acute pancreatitis

\section{Background}

Acute pancreatitis is a frequently appreciated severe disease and can be associated with the spreading of the inflammatory process and leakage of the pancreatic fluid [1]. The mortality rate in mild acute pancreatitis is low [2], but unfortunately, $20 \%$ of the patients may advance to acute severe pancreatitis that may have a fast onset, multiple organ dysfunctions and/or failure, and also high mortality rate [3]. Prompt diagnosis of acute pancreatitis

\footnotetext{
* Correspondence: tamirhaq@yahoo.com

Faculty of Medicine, Radiology Department, Zagazig University, Zagazig, Egypt
}

\section{SpringerOpen}

severity is vital for ideal management planning to decrease the potential mortality.

The pancreas lacks fascial surface covering and is situated in the anterior pararenal space. Inflammatory pancreatic fluid can infiltrate the retroperitoneal, abdominal, and pelvic cavities when pancreatitis occurs [4]. Usually, the retroperitoneal cavity is divided into three parts: the anterior pararenal, posterior pararenal, and perirenal spaces. They are demarcated by the prerenal fascia, lateroconal fascia, and posterior renal fascia [5].

The interfascial planes were offered by Molmenti et al. [6] as an anatomical model of the retroperitoneal space,
( ) The Author(s). 2020 Open Access This article is licensed under a Creative Commons Attribution 4.0 International License, which permits use, sharing, adaptation, distribution and reproduction in any medium or format, as long as you give appropriate credit to the original author(s) and the source, provide a link to the Creative Commons licence, and indicate if changes were made. The images or other third party material in this article are included in the article's Creative Commons licence, unless indicated otherwise in a credit line to the material. If material is not included in the article's Creative Commons licence and your intended use is not permitted by statutory regulation or exceeds the permitted use, you will need to obtain permission directly from the copyright holder. To view a copy of this licence, visit http://creativecommons.org/licenses/by/4.0/. 
proposing that each retroperitoneal fascia is formed of multiple distinct layers with potential space between these layers of fascia; thus, Molmenti et al. [6] divided the retroperitoneal space into 9 parts: three spaces (anterior pararenal, posterior pararenal, and perirenal spaces) and 6 interfascial planes (retromesenteric, lateroconal interfascial, retrorenal, combined interfascial planes, prevesical, and presacral spaces). The spread of the inflammatory fluid during the pancreatitis process within the retroperitoneal space may be better explained using interfascial planes. Ishikawa et al. [7] proposed in addition to the previous classification another subfascial plane situated between the posterior pararenal space and transversalis fascia, and this plane is connected by a narrow passage to the retrorenal plane. Ishikawa et al. [7] found the CT-based classification of acute pancreatitis based on retroperitoneal extension with the idea of subfascial planes as a useful indicator of disease severity and prognosis [8].

For the prediction of the severity of acute pancreatitis, many clinical scoring systems, e.g., the Ranson score or the Acute Physiology \& Chronic Health Evaluation II score (APACHE II) have been used [9]. Also, Balthazar et al. [9] established a CT-based classification system for acute pancreatitis for a combined evaluation of the extent of pancreatic or extrapancreatic inflammation and the pancreatic necrosis extent $[7,8,10]$.

Balthazar and his colleagues [10] developed the CT severity index which is an important improvement as it helps clinicians to distinguish mild, moderate, and severe types of acute pancreatitis by focusing on the incidence and degree of pancreatic inflammation and necrosis. The scoring system composed of 10-point severity scale, and each point is marked for the presence or absence of fluid collections, assessment of the presence, and the degree of necrosis of the pancreatic tissue on contrast enhanced CT [10]. Unfortunately, this index has some limitations. First, no significant correlation between the score achieved by the CT severity index and subsequent development of organ failure [11], parenchymal extrapancreatic complications $[4,12]$, or the vascular complications in the peripancreatic area [13]. The second limitation was only moderate inter-observer agreement in scoring CT images using the CT severity index $[4,14,15]$.

Koenraad et al. [15] developed the modified CT scoring severity index (CTSI) in acute pancreatitis and tried to overcome these limitations to predict clinical outcomes better than the CT severity index, keeping or better improving the inter-observer agreement.

The aim of this study is to compare the results of the modified CT severity index and retroperitoneal interfascial extension CT grading system in the grading of the severity of acute pancreatitis and compare both grading systems to the clinical course of the disease and its complications

\section{Methods \\ Patients}

This prospective study was conducted between January 2015 and June 2018 in the tertiary center and included 50 patients, (40 males and 10 females) with a mean age of $52.12 \pm 2.0$ years (range, 27-90 years), all presented with acute pancreatitis and admitted to the intensive care unit 1-3 days after the onset of the symptoms. All patients gave their informed consent, and this study was approved by the local institutional review board.

Exclusion criteria included patients with traumatic pancreatitis, a renal impairment that hinders in performing contrast-enhanced CT, and patients with a history of laparotomy or with a previous hospitalization for pancreatitis.

\section{CT Technique}

All patients were scanned by Somatom Balance (Siemens Medical Solutions, Germany) helical CT machine. Contrast-enhanced CT scans (reconstruction thickness: $5 \mathrm{~mm}$, collimation: $4 \times 2.5 \mathrm{~mm}$, reconstruction intervals: $5 \mathrm{~mm}$ ) were obtained with scan delay $40 \mathrm{~s}$ after IV administration of $100 \mathrm{~mL}$ of iopromide $300 \mathrm{mg} \mathrm{I} / \mathrm{mL}$ (Ultravist 300, Berlex Laboratories), injected in 18 gage cannula in the ante-cubital vein with an injection of 3.0 $\mathrm{mL} / \mathrm{s}$ using an automatic power injector. Oral administration of $1 \mathrm{~L}$ of water mixed with $20 \mathrm{~mL}$ of gastrografin $90 \mathrm{~min}$ before the exam for opacification of the bowels was done.

\section{Image analysis of CT scans}

Images were transferred to the PACS workstations (Philips, iSite radiology) and independently reviewed by two qualified abdominal radiologists who were uninformed about the patient clinical data. Each radiologist assessed the severity of pancreatitis for each patient using modified CT severity index parameters shown in Table 1.

This modified index differs from the old CT severity index in the simplified evaluation of the presence/number of fluid collections, the extent of pancreatic necrosis, and the evaluation of extrapancreatic findings, e.g., ascites, pleural fluid, parenchymal extrapancreatic abnormalities (hemorrhage, infarction, or sub-capsular fluid collection), involvement of the GIT (inflammation, intramural fluid collection, or perforation), and vascular complications (arterial hemorrhage, venous thrombosis, or pseudo-aneurysms). Using the modified index, the pancreatitis severity for each patient was graded into mild (0-2 points), moderate (4-6 points), or severe (8-10 points) [15]. 
Table 1 Modified CT severity index parameters quoted by Mortele et al. [4]

\begin{tabular}{ll}
\hline Modified CT severity index & Points \\
\hline Prognostic indicators & \\
Inflammation of the pancreas & 0 \\
$\quad$ Normal pancreas & 2 \\
$\begin{array}{l}\text { Intrinsic abnormalities in the pancreas with/without } \\
\text { peripancreatic fat inflammatory changes }\end{array}$ & \\
$\begin{array}{l}\text { Peripancreatic fat necrosis or pancreatic or peripancreatic fluid } \\
\text { collection }\end{array}$ & 4 \\
Pancreatic tissue necrosis & 0 \\
$\quad \begin{array}{l}\text { None } \\
\leq 30 \%\end{array}$ & 2 \\
$>30 \%$ & 4 \\
Extrapancreatic complications & \\
$\begin{array}{l}1 \text { or more of ascites, pleural effusion, parenchymal, or vascular } \\
\text { or GlT complications }\end{array}$ & \\
\hline
\end{tabular}

Each patient was graded by modified CT severity index grading score and the retroperitoneal interfascial plane grading system developed by Ishikawa et al. [7] grading system (summarized in Fig. 1) during the same interpretation session as the aim of the work was to correlate these two grading systems and doing this by the same radiologist at the same session could minimize the potential bias that could happen if the same reviewer grades the same extent of inflammation differently with the two grading systems.

\section{Grading of acute pancreatitis according to Ishikawa et al. [7] grading system}

The distribution of inflammatory fluid collection in different retroperitoneal component parts is summarized in Table 2.

- Grade I (Fig. 2): fluid collection is localized to the anterior pararenal space or spreads to the retromesenteric plane adjacent to the anterior pararenal space.

- Grade II (Fig. 3): the fluid spreads more into the lateroconal plane or retrorenal plane through the retromesenteric plane

- Grade III (Fig. 4): the fluid spreads more inferiorly to the combined interfascial plane

- Grade IV (Fig. 4): the fluid escapes from the interfascial plane through the narrow connecting pathway to the subfascial plane.

- Grade V (Fig. 5): the fluid infiltrates directly into the posterior pararenal space across the subfascial plane, lateroconal, or retrorenal plane. The fluid collection in the pelvic prevesical and presacral spaces was found in grade V (Fig. 5).

- The inflammatory fluid collection can infiltrate into the perirenal space (Fig. 1a) from the interfascial
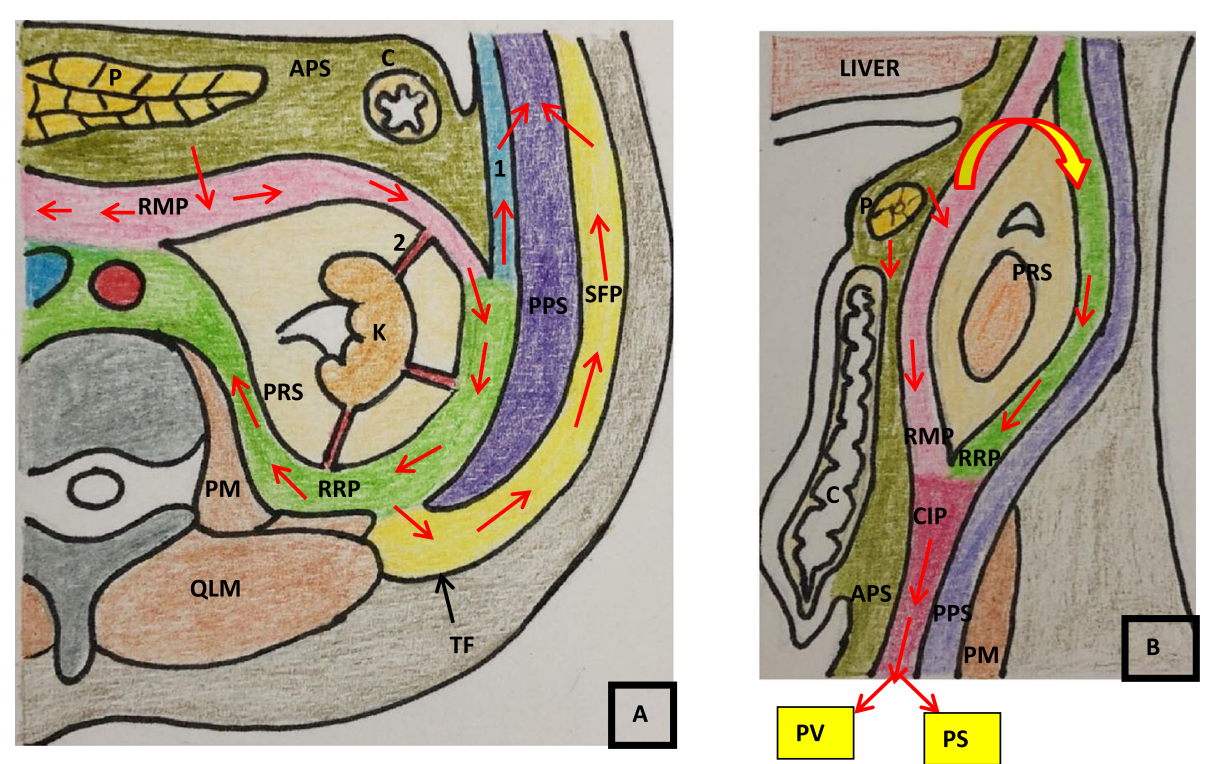

Fig. 1 Retroperitoneal illustrative drawings showing the pathway of the spread of acute pancreatitis. a Cross-sectional drawing at the level of the left kidney showing the retroperitoneal spaces: the anterior pararenal space (APS), perirenal space (PRS), and posterior pararenal space (PPS). The interfascial planes: the retromesenteric plane (RMS), retrorenal plane (RRP), and lateroconal plane (1). A narrow connecting the passage way communicates the subfascial plane with the retrorenal plane. Red arrows show the retroperitoneal pathway of spread of acute pancreatitis. $\mathbf{b}$ Sagittal drawing shows the combined interfascial plane (CIB) continues into the prevesical space (PV) and presacral space (PS) in the pelvis. Abbreviations: K: kidney, PM: psoas muscle, QLM: quadratus lumborum muscle, SFP: subfascial plane, TF: transversalis fascia, C: colon. Quoted and edited from [7] 
Table $\mathbf{2}$ CT detection of the distribution of the acute fluid collection in the interfascial planes

\begin{tabular}{|c|c|c|c|c|c|c|c|c|c|}
\hline Grade & Patients subdivisions & APS & RMP & LCP, RRP & $\mathrm{CIP}$ & SFP & PPS & PRS & PV, PS \\
\hline \multirow[t]{2}{*}{ G I $(n=7)$} & 1 & $\sqrt{ }$ & $x$ & $x$ & $x$ & $x$ & $\mathrm{X}$ & $\mathrm{X}$ & $\mathrm{X}$ \\
\hline & 6 & $\sqrt{ }$ & $\sqrt{ }$ & $x$ & $x$ & $x$ & $\mathrm{x}$ & $\sqrt{ }$ & $x$ \\
\hline \multirow[t]{2}{*}{ G II $(n=14)$} & 7 & $\sqrt{ }$ & $\sqrt{ }$ & $\sqrt{ }$ & $\mathrm{X}$ & $\mathrm{X}$ & $x$ & $\mathrm{X}$ & $\mathrm{x}$ \\
\hline & 7 & $\sqrt{ }$ & $\sqrt{ }$ & $\sqrt{ }$ & $x$ & $x$ & $\mathrm{x}$ & $\sqrt{ }$ & $x$ \\
\hline \multirow[t]{2}{*}{ GIII $(n=7)$} & 3 & $\sqrt{ }$ & $\sqrt{ }$ & $\sqrt{ }$ & $\sqrt{ }$ & $x$ & $\mathrm{x}$ & $\mathrm{X}$ & $x$ \\
\hline & 4 & $\sqrt{ }$ & $\sqrt{ }$ & $\sqrt{ }$ & $\sqrt{ }$ & $x$ & $\mathrm{x}$ & $\sqrt{ }$ & $x$ \\
\hline \multirow[t]{2}{*}{ G IV $(n=13)$} & 2 & $\sqrt{ }$ & $\sqrt{ }$ & $\sqrt{ }$ & $x$ & $\sqrt{ }$ & $\mathrm{x}$ & $\mathrm{x}$ & $x$ \\
\hline & 11 & $\sqrt{ }$ & $\sqrt{ }$ & $\sqrt{ }$ & $\sqrt{ }$ & $\sqrt{ }$ & $\mathrm{X}$ & $\sqrt{ }$ & $\mathrm{x}$ \\
\hline \multirow[t]{2}{*}{$\mathrm{GV}(n=9)$} & 7 & $\sqrt{ }$ & $\sqrt{ }$ & $\sqrt{ }$ & $\sqrt{ }$ & $\sqrt{ }$ & $\sqrt{ }$ & $\sqrt{ }$ & $x$ \\
\hline & 2 & $\sqrt{ }$ & $\sqrt{ }$ & $\sqrt{ }$ & $\sqrt{ }$ & $\sqrt{ }$ & $\sqrt{ }$ & $\sqrt{ }$ & $\sqrt{ }$ \\
\hline
\end{tabular}

$\sqrt{ }$ indicates the fluid collection was detected, and $\mathbf{X}$ indicates the fluid collection was not detected. Note different colors for different grades parallel to the limit of extension

APS anterior pararenal space, $L C P$ lateroconal plane, $R M P$ retromesenteric plane, CIP combined interfascial plane, $R R P$ retrorenal plane, SFP subfascial plane, $P R S$ perirenal space, $P P S$ posterior pararenal space, $P V$ prevesical space, $P S$ presacral space

planes through perinephric bridging septa in each grade.

\section{Patients outcome parameters}

Patients' outcome parameters were collected from the HIS/RIS systems (hospital and radiology information systems) including the length of the hospital stay (counted in days), evidence of infection (positive Gram stain or culture or the combination of increased WBC > $15,000 / \mathrm{mm}^{3}$ and fever $>100{ }^{\circ} \mathrm{F}$ ), need for intervention (percutaneous aspiration and or drainage or surgical intervention), and evidence of organ failure. Patient medical charts were reviewed for the occurrence or absence of dysfunction in 6 separate systems, i.e., for respiratory system failure, cardiovascular system, renal, central nervous system, hepatic, or hematologic system failures as defined by Fagon et al. [16].

\section{Data analysis}

Values are presented as a number or percentage, except the mechanical ventilation and the duration of ICU stay which are expressed as mean \pm standard error of the mean. The variances in the clinical data in-between the grades were analyzed by one-way factorial analysis of variance by the post hoc Scheffe method and a $p$ value of $<0.05$ was considered significant statistically.

The inter-observer agreement for assessing the severity of pancreatitis with both the modified CT severity index and retroperitoneal extension grading system was calculated. The percentage of agreement as well as the kappa statistic was used to calculate the proportion of interpreter agreement above the expected by chance.

A weighted kappa statistic of $0.41-0.60$ was considered as moderate agreement, $0.61-0.80$ was taken as good agreement, and 0.81-1.00 was taken as excellent
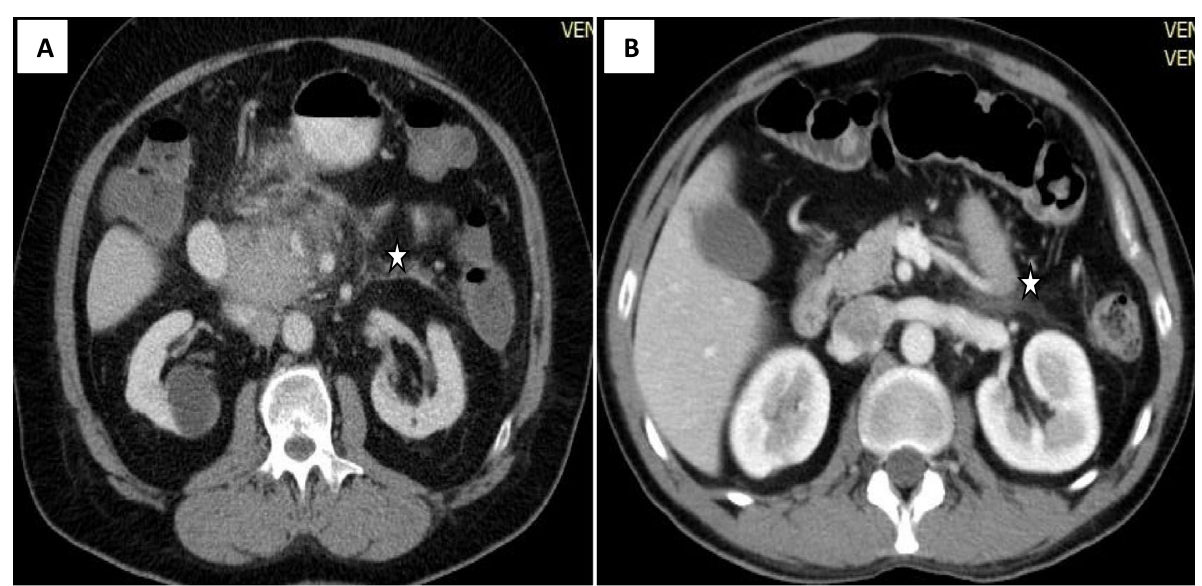

Fig. 2 Grade I acute pancreatitis with modified CTSI (mild score 4). a CT scan at the level of the kidney shows fluid collection spreading into the retromesenteric plane (white star). $\mathbf{b}$ CT scan of different patients at the level of the kidney shows fluid collection spreading into the retromesenteric plane (white star) 

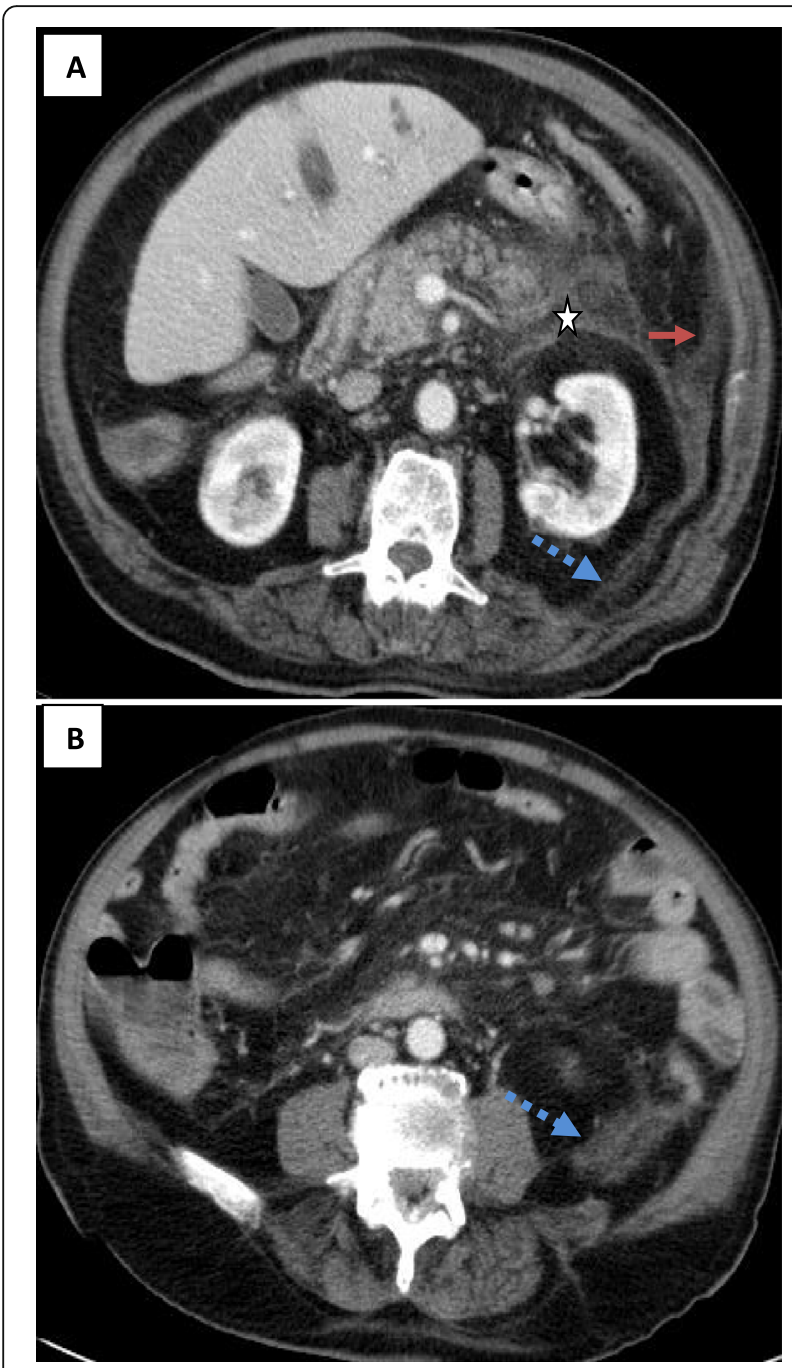

Fig. 3 Grade II acute pancreatitis with modified CTSI ( mild score 4). $\mathbf{a}, \mathbf{b}$ CT scan at the level of the kidney (a) and at a lower level (b) shows fluid collection spreading into the retromesenteric plane (white star) extending into the lateroconal plane (red arrow) and retro-renal plane (dotted blue arrow)

agreement [17]. In case of controversy between the two observers, a conjoint meeting was done and solved by consensus.

\section{Results}

This study included 50 patients, all of them presented with acute pancreatitis and admitted to the intensive care unit; the causes of pancreatitis were due to gallstones in 29, alcohol abuse in 3 patients, post ERCP inflammation in 9, and miscellaneous or unknown etiology in 9 patients. Forty-eight patients (96\%) were initially clinically diagnosed upon admission as having severe acute pancreatitis according to the clinical Atlanta classification system [18].
Relation between retroperitoneal extension grading system and the clinical course

The relation between $\mathrm{CT}$ grades and clinical course is summarized in Table 3. Among the 22 patients with grades IV and V, acute pancreatitis was complicated by respiratory insufficiency in 17 patients (77.3\%), organ failure in 11 patients (50\%), abscess in 8 patients (36.4\%), and ARF in 7 patients (31.8\%). The duration of the ICU stay and mechanical ventilation if applicable was significantly longer for these 22 patients than for patients with a lower grade (I, II, or III diseases). In 41 out of 50 patients $(82 \%)$, the acute fluid collection resolved spontaneously. In 9 patients (18\%), 1 with grade II, 3 with grade IV, and 5 with grade $\mathrm{V}$ pancreatitis, fluid collection progressed to abscess formation and requires drainage (percutaneous or open drainage)) about $24.2 \pm$ 3.4 days after initial admission. Nineteen patients (86.4\%) had morbidity for grade IV and V pancreatitis, and 6 of them unfortunately died (2 died from fulminant disease about 9 days of admission, and the other 4 died from organ failure more than 21 days after admission).

\section{Relation between modified CTSI and the clinical course}

The relation between modified CTSI and clinical course is summarized in Table 4. Among the 35 patients with moderate (4-6 points) and severe (8-10 points) disease, pancreatitis was complicated by respiratory insufficiency in 20 patients $(57.1 \%)$, organ failure in 11 patients (31.4\%), abscess in 9 patients (25.7\%), and ARF in 8 patients $(22.9 \%)$. The length of ICU stay was significantly longer for these 35 patients than for patients with mild (0-2 points) disease. Twenty-two patients (6.28\%) had morbidity for moderate/severe grades, and 6 of them died (17.1\%), 3 with moderate disease and 3 with severe disease.

\section{Relation between retroperitoneal extension grading and the modified CTSI}

The relation between the interfascial grading system and modified CTSI scores is summarized in Table 5. According to the modified CTSI, 15 patients (30\%) had mild pancreatitis, 25 (50\%) had moderate pancreatitis, and 10 (20\%) had severe pancreatitis. All patients underwent contrast-enhanced CT as we excluded from the start patient with renal impairment, so all patients had been assessed for pancreatic gland necrosis as well as extrapancreatic complications including vascular complications. Splenic vein thrombosis was found in 2 patients with severe pancreatitis (Fig. 5). Only 3 patients with mild pancreatitis show morbidity, and the other 12 patients had a very good outcome without any serious local complication; this was correlated to grades I and II as only 3 patients with grade II (out of 21 patients in both grades) show morbidity, and this was considered as a 

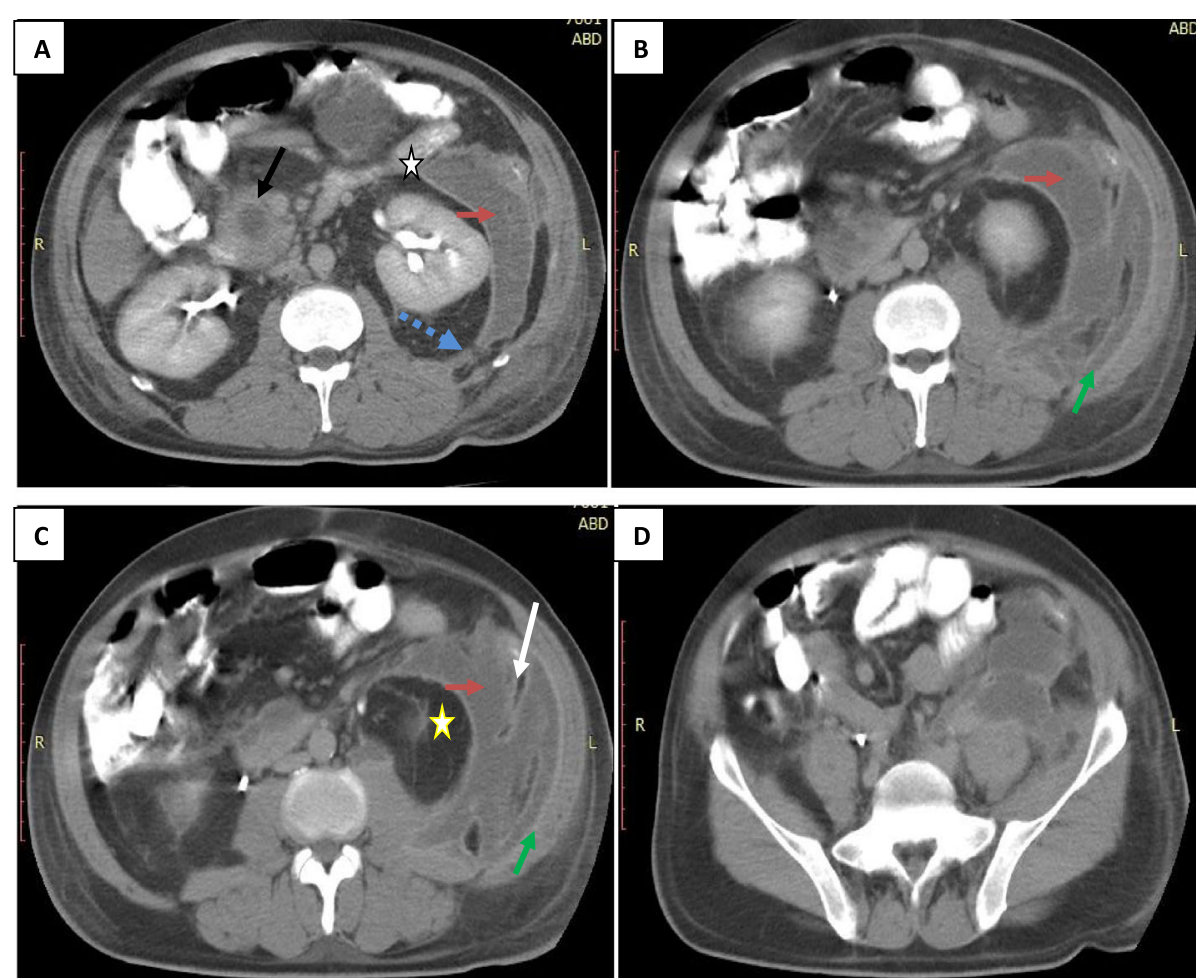

Fig. 4 Grade III acute pancreatitis with modified CTSI (moderate score 6). a-d CT shows acute fluid collection spreading from the retromesenteric plane (white star), lateroconal plane (red arrow), and retrorenal plane (dotted blue arrow) into the combined interfascial plane (yellow star) and infiltrating the posterior pararenal space (white arrow) with pancreatic pseudo-cyst seen (black arrow). Further extension of the fluid seen at a lower level beyond the interfascial plane into the subfascial plane (green arrow) occurred within 9 days, so the case progresses to grade IV within 9 days (the modified CTSI progresses to severe score 10)

mild disease. Four patients with moderate pancreatitis (score 4) were graded as grades I or II (Figs. 2 and 3). Mortality in the 35 patients with moderate and severe pancreatitis was $6(17.1 \%)$ out of 35 patients, and these were comparable to the combined grade IV and $\mathrm{V}$ patients (total of 22 with 6 patients (27.3\%) who show mortality and this was considered as a severe disease. The 25 patients with a modified CT score of moderate pancreatitis had poor outcomes: 13 patients (52\%) had a complication, and 3 patients (12\%) died. Of these 25 patients, 20 with grade III and IV disease had a poor outcome; morbidity was $65 \%$ and mortality was $10 \%$ and this was considered as a moderate disease.

Both grading systems show a statistical significant correlation in terms of the length of hospital stay $(p=$ 0.0324 for the modified CTSI and $p=0.301$ for the inter-fascial plane grading), the need for surgical or percutaneous interventions with both modified CTSI $(p=$ $0.0324)$ and the inter-fascial plane grading $(p=0.0112)$, and the development of organ failure.

The inter-observer agreement for evaluating the severity of pancreatitis using both the grading systems using weighted kappa statistic was 0.83 indicating excellent agreement

\section{Discussion}

The CT severity index was introduced in 1994 for assessment of the severity of acute pancreatitis [10] and was accepted internationally but had some limitations $[4,11-14]$ including the assessment of extrapancreatic parenchymal complications, the presence of organ failure [4, 12], and peripancreatic vascular complications [14]. Another limitation was the lack of significant difference in morbidity and mortality between patients who have $30-50 \%$ pancreatic necrosis and those who have $>50 \%$ necrosis. Owing to these limitations, a simplified modified CT severity index offered by Koenraad et al. [15] was adopted to overcome these limitations.

Ishikawa et al. [7] was the first to grade the severity of acute pancreatitis according to its retroperitoneal spread using the concept of interfascial plane extension and the uniqueness in Ishikawa et al. [7]'s study that they found a passageway of spread beyond the interfascial planes, thus succeeded in categorizing all forms of fluid collection in the retroperitoneum into 5 grades.

Both classification systems utilize the natural spread of the disease and recognize the severity 

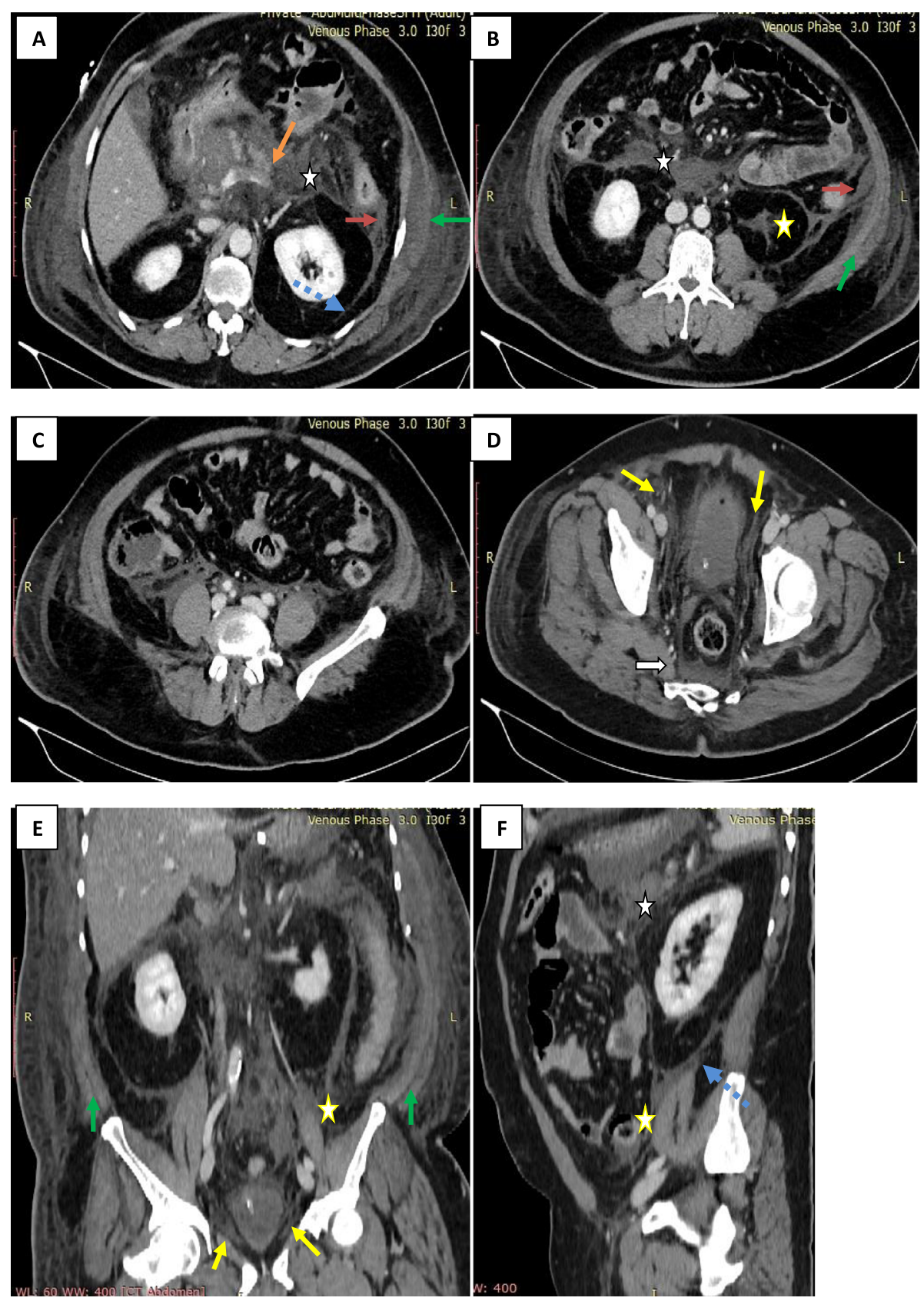

Fig. 5 Grade V acute pancreatitis with modified CTSI (severe score 10). a CT scan shows almost more than 30\% of the necrotic pancreatic tissue with the thrombosed splenic vein (yellow arrow), fluid collection in the retromesenteric plane (white star in $\mathbf{a}$, $\mathbf{b}$, and $\mathbf{f}$ ), lateroconal plane (red arrow), and retrorenal plane (dotted blue arrow). Further, the pancreatic inflammation extends into both subfascial planes (green arrow in $\mathbf{b}$ and e). The retromesenteric plane and retrorenal plane have merged into the combined interfascial planes (yellow star in $\mathbf{b}, \mathbf{e}$, and $\mathbf{f}$ ). $\mathbf{c}$, $\mathbf{d}$ CT scan at a lower level shows the caudal extension of the fluid collection reaching the prevesical space (yellow arrows in $\mathbf{d}$ and $\mathbf{e}$ ) and presacral space (white filled arrow in $\mathbf{d}$ ) via the combined interfascial plane. $\mathbf{f}$ Sagittal view shows clearly the combined interfacial plane (yellow asterisk). This patient died from multiple organ failure dysfunction syndrome

and the prognosis of the disease better than the CTSI because it is not accurate enough to represent the location of the retroperitoneal lesions as it is based on the old theory of the retroperitoneal structure [5].
In our study, we tried to correlate both grading systems and we found that modified CTSI requires intravenous (IV) contrast injection to define pancreatic necrosis and extrapancreatic complications like vascular complications which indeed important prognostic 
Table 3 Relation between interfascial plane CT grading system and the clinical course

\begin{tabular}{|c|c|c|c|c|c|}
\hline \multirow[t]{3}{*}{ Clinical course } & \multicolumn{5}{|l|}{$\mathrm{CT}$ grade } \\
\hline & Grade I & Grade II & Grade III & Grade IV & Grade V \\
\hline & $(n=7)$ & $(n=14)$ & $(n=7)$ & $(n=13)$ & $(n=9)$ \\
\hline ARF & $x$ & $1(7.1 \%)$ & $x$ & $3(23.1 \%)$ & $4(44.4 \%)$ \\
\hline Respiratory insufficiency & $x$ & $x$ & $3(42.9 \%)$ & $8(61.5 \%)$ & $9(100 \%)$ \\
\hline Abscess & $x$ & $1(7.1 \%)$ & $x$ & $3(23.1 \%)$ & $5(55.6 \%)$ \\
\hline Organ failure & $x$ & $x$ & $x$ & $4(30.8 \%)$ & $7(77.8 \%)$ \\
\hline Average ICU length of stay (days) & $3 \pm 0.5$ & $3.4 \pm 0.6$ & $7.5 \pm 1.1$ & $25.1 \pm 4.2$ & $32.1 \pm 8.6$ \\
\hline Drainage (PD/OD) of abscess or pseudo-cyst & $x$ & $1(\mathrm{PD})$ & $x$ & $3(2 \mathrm{PD}, 1 \mathrm{OD})$ & 5 (4 PD, $1 \mathrm{OD})$ \\
\hline Morbidity & $x$ & $3(21.4 \%)$ & $3(42.6 \%)$ & 10 (76.9\%) & 9 (100\%) \\
\hline Mortality & $x$ & $x$ & $x$ & 2 (15.4\%) & $4(44.4 \%)$ \\
\hline
\end{tabular}

$A R F$ acute renal failure, $I C U$ intensive care unit, $P D$ percutaneous drainage, $O D$ open drainage, $N . B$ some patients had more than one clinical course

factors that is why we excluded patients with contraindication to contrast administration. Therefore, we searched for another prognostic indicator that does not depend on contrast injection as a complement to the modified CTSI. We applied Ishikawa et al. [7] grading system for the study group patients, and we faced no difficulty in assessing the extension of inflammatory fluid.

The correlation between the two grading system reveals a significant correlation in detecting morbidity related to the disease in mild pancreatitis (scores $0-2$ ) that was correlated to grades I and II and was considered as a mild disease, while 4 patients with moderate pancreatitis (score 4) were graded as grades I or II; thus, score 4 can be considered as early moderate pancreatitis as these cases resolved spontaneously after medical treatment. Both grading systems show a strong correlation in terms of mortality in the moderate and severe pancreatitis (scores $8-10)$ that was comparable to the combined grades (IV and V) and was considered as a severe disease. Both grading systems show a good correlation in patients with modified CTSI of moderate pancreatitis (scores 4-6) and with grade III and IV pancreatitis in terms of morbidity and mortality, and this was considered as a moderate disease in agreement with the results of $[7,15]$ in each grading system.

Both grading systems show a statistical significant correlation in terms of the length of hospital stay, the need for surgical or percutaneous interventions, and the development of organ failure in agreement with the results of $[7,15]$ in each grading system.

We think that we can apply Ishikawa et al. [7]'s grading system as a prognostic indicator if there is a contraindication to contrast administration as it does not depend on contrast-enhanced CT.

In the present study, fluid collection confined to the interfascial planes, corresponding to (grade I, II, or III) pancreatitis, resolved spontaneously in all cases (except for one patient with grade II), while inflammatory fluid collection in 3 patients with grade IV disease and in 5 patients with grade $\mathrm{V}$ disease needed drainage in agreement with Ishikawa et al. [7] who mentioned that the fluid in the interfascial planes may drain spontaneously while for grades IV and V, the persistent fluid collection can easily progress to infectious abscess requiring drainage.

In the current study, we did not analyze the ventral extension of pancreatic inflammatory fluid, e.g., into

Table 4 Relation between modified CTSI score and the clinical course

\begin{tabular}{llll}
\hline Clinical course & \multicolumn{2}{l}{ Modified CTSI score } \\
\cline { 2 - 4 } & Mild (0-2 points) $(n=15)$ & Moderate (4-6 points) $(n=25)$ & Severe $(8-10$ points) $(n=10)$ \\
\hline ARF & $X$ & $4(16 \%)$ & $4(40 \%)$ \\
Respiratory insufficiency & $X$ & $10(40 \%)$ & $10(100 \%)$ \\
Abscess & $X$ & $4(16 \%)$ & $5(50 \%)$ \\
Organ failure & $X$ & $6(24 \%)$ & $5(50 \%)$ \\
Average ICU length of stay (days) & $3.5 \pm 0.5$ & $15.7 \pm 2.9$ & $31.3 \pm 6.1$ \\
Drainage (PD/OD) of abscess or pseudo-cyst & $1(6.7 \%)$ & $6(24 \%)$ & $2(20 \%)$ \\
Morbidity & $3(30 \%)$ & $13(52 \%)$ & $9(90 \%)$ \\
Mortality & $X$ & $3(12 \%)$ & $3(30 \%)$
\end{tabular}


Table 5 Correlation between modified CTSI score and interfascial plane grading system

\begin{tabular}{|c|c|c|c|c|}
\hline \multirow{2}{*}{$\begin{array}{l}\mathrm{CT} \text { interfascial } \\
\text { plane extension } \\
\text { grading system }\end{array}$} & \multicolumn{4}{|l|}{ Modified CTSI score } \\
\hline & Mild (0-2 points) $(n=15)$ & Moderate (4-6 points) $(n=25)$ & Severe (8-10 points) $(n=10)$ & Total \\
\hline Grade I $(n=7)$ & $7(46.7 \%)$ & $x$ & $x$ & 7 \\
\hline Grade $\|(n=14)$ & $8(53.3 \%)$ & $6(24 \%)$ & $x$ & 14 \\
\hline Grade III $(n=7)$ & $x$ & $6(24 \%)$ & $1(10 \%)$ & 7 \\
\hline Grade IV $(n=13)$ & $x$ & $8(32 \%)$ & $3(30 \%)$ & 11 \\
\hline Grade $\vee(n=9)$ & $x$ & $5(20 \%)$ & $6(60 \%)$ & 11 \\
\hline Total $(n=50)$ & 15 & 25 & 10 & 50 \\
\hline
\end{tabular}

the transverse mesocolon or the lesser sac, and this can be explained by that the fluid collection in these locations often observed in every grade as this fluid results from a direct extension or leakage from the anterior pararenal space in agreement with $[6,7,18-$ 20] studies who reported that local complication, e.g., pseudocyst or pancreatic abscess can develop in these locations with no definite established relation between retroperitoneal grading system and the degree of ventral extension of acute pancreatitis or pancreatic necrosis.

In the current study, we overcome this limitation to some extent by applying modified CTSI as extrapancreatic complications can successfully be recorded as well as peripancreatic collection; thus, we recommend a combined grading system evaluation whenever possible.

Evaluation of the patients by the same radiologist at the same setting in this study decreased the bias; thus, the inter-observer agreement in assessing the severity of pancreatitis was an excellent agreement according to Fleiss et al. [17]'s criteria.

This study had some important limitations. First, it was done only with contrast-enhanced CT that represents bias as many patients with acute pancreatitis were excluded from the study due to renal impairment, but our aim was to standardize the protocol in both grading systems to know the outcome. Second, it has a small sample size owing to narrow selection criteria. This can be explained by that we performed CT within 1-3 days after the onset of the symptoms aiming to establish the correct prognostic value of both grading systems in acute pancreatitis at the time of presentation, and this leads to a small number of patients and narrow selection criteria because not all patients with acute pancreatitis need to have a CT examination.

To our limited knowledge, this is the first work to correlate combined modified CTSI and interfacial plane grading in the grading of acute pancreatitis and we think that a larger number of patients in multicenter trials need to be conducted for further assessment.

\section{Conclusion}

In conclusion, a good correlation was observed between the modified CTSI and retroperitoneal extension grading systems in grading the severity of acute pancreatitis. We think that we can apply Ishikawa et al.'s grading system as a prognostic indicator if there is a contraindication to contrast administration.

\section{Abbreviations}

APS: Anterior pararenal space; RMP: Retromesenteric plane; LCP: Lateroconal plane; RRP: Retrorenal plane; CIP: Combined interfascial plane; SFP: Subfascial plane; PPS: Posterior pararenal space; PRS: Perirenal space; PS: Presacral space; PV: Prevesical space

\section{Acknowledgements}

No acknowledgements.

\section{Authors' contributions}

$\mathrm{TH}$ conceived of the study, participated in its design and coordination, drafted the manuscript, and carried out radiological results. NM participated in the design of the study and sequence alignment and in radiological/ surgical correlation and helped in drafting the results. The authors have read and approved the final manuscript.

\section{Funding}

No funding sources.

\section{Availability of data and materials}

The datasets used and analyzed during the current study are available from the corresponding author on reasonable request.

\section{Ethics approval and consent to participate}

The study was approved by the ethical committee of the Faculty of Medicine, Zagazig University, and an informed written consent to participate in the study was taken from all subjects. The date of the approval of the protocol was Jan 2015 from the Department of Radiology, Faculty of Medicine, Zagazig University. The committee has no reference number, only the date.

\section{Consent for publication}

All patients gave a written informed consent for publication of the study. If the patient was decreased consciousness or comatose, written informed consent was given by the legal guardians.

Competing interests

The authors declare that they have no competing interests. 
Received: 13 April 2020 Accepted: 13 May 2020

Published online: 21 May 2020

\section{References}

1. Schepers NJ, Besselink MG, Van Santvoort HC, Bakker OJ, Bruno MJ (2013) Dutch Pancreatitis Study Group. Early management of acute pancreatitis. Best Pract Res ClinGastroenterol 27:727-743

2. Russo MW, Wei JT, Thiny MT, Gangarosa LM, Brown A, Ringel Y, Shaheen NJ, Sandler RS (2004) Digestive and liver diseases statistics, 2004. Gastroenterology 126:1448-1453

3. Banks PA, Freeman ML (2006) Practice Parameters Committee of the American College of Gastroenterology. Practice guidelines in acute pancreatitis. Am J Gastroenterol 101:2379-2400

4. Mortelé KJ, Mergo PJ, Taylor HM, Ernst MD, Ros PR (2000) Renal and perirenal space involvement in acute pancreatitis: spiral $C T$ findings. Abdom Imaging 25:272-278

5. Meyers MA (2000) The extraperitoneal spaces: normal and pathologic anatomy. In: Meyers MA (ed) Dynamic radiology of the abdomen: normal and pathologic anatomy. Springer-Verlag, New York, pp 333-492

6. Molmenti EP, Balfe DM, Kanterman RY, Bennett HF (1996) Anatomy of the retroperitoneum: observations of the distribution of pathologic fluid collections. Radiology 200:95-103

7. Ishikawa K, Idoguchi K, Tanaka H, Tohma Y, Ukai I, Watanabe H, Matsuoka T, Yokota J, Sugimoto T (2006) Classification of acute pancreatitis based on retroperitoneal extension: application of the concept of interfascial planes. Eur J Radiol 60:445-452

8. Chi X-X, Chen T-W, Huang X-H, Lin Y, Tang W et al (2016) Magnetic resonance imaging of retroperitoneal interfascial plane involvement in acute pancreatitis. Quant Imaging Med Surg 6(3):250-258

9. Kumar AH, Griwan MS (2018) A comparison of APACHE II, BISAP, Ranson's score and modified CTSI in predicting the severity of acute pancreatitis based on the 2012 revised Atlanta Classification. Gastroenterology Report 6(2):127-131

10. Chatzicostas C, Roussomoustakaki M, Vardas E, Romanos J et al (2003) Balthazar computed tomography severity index is superior to Ranson criteria and APACHE II and III scoring systems in predicting acute pancreatitis outcome. J ClinGastroenterol 36:253-260

11. Lankish PG, Pflichthofer D, Lehnick D (2000) No strict correlation between necrosis and organ failure in acute pancreatitis. Pancreas 20:319-322

12. Wiesner W, Studler U, Kocher T, Degen L, Buitrago Tellez CH, Steinbrich W (2003) Colonic involvement in non-necrotizing acute pancreatitis: correlation of $C T$ findings with the clinical course of affected patients. EurRadiol 13:897-902

13. Inoue K, Hirota M, Beppu T et al (2003) Angiographic features in acute pancreatitis: the severity of abdominal vessel ischemic change reflects the severity of acute pancreatitis. J Pancreas 4:207-213

14. Lecesne R, Tourel P, Bret PM et al (1999) Acute pancreatitis: interobserver agreement and correlation of CT and MR cholangiopancreatography with outcome. Radiology 211:727-735

15. Koenraad J. Mortele, Walter Wiesner, Lisa Intriere, Shridhar Shankar, et al. (2004). A modified CT severity index for evaluating acute pancreatitis: improved correlation with patient outcome. AJR; 183, November: 1261-1265

16. Fagon JY, Chastre J, Novara A, Medioni P, Gibert C (1993) Characterization of intensive care unit patients using a model based on the presence or absence of organ dysfunctions and/or infection: the ODIN model. Intensive Care Med 19:137-144

17. Fleiss JL (1981). Statistical methods for rates and proportions, New York, NY: Wiley; 2nd edition: 212-255

18. Bradley EL 3rd (1993) A clinically based classification system for acute pancreatitis. Summary of the international symposium on acute pancreatitis, Atlanta, GA, September 11-13, 1992. Arch Surg 128:586-590

19. Balfe DM, Molmenti EP, Bennett HF (1998). Normal abdominal and pelvic anatomy. In: Lee JK, Sagel SS, Stanley RJ, Heiken JP, editors. Computed body tomography with MRI correlation. Philadelphia, PA: Lippincott Williams \& Wilkins; 3rd ed:573-636.

20. Kazuo I, Nakao S, Nakamuro M, Huang T-P, Nakano H (2016) The retroperitoneal interfascial planes: current overview and future perspectives. Acute Medicine \& Surgery 3:219-229

\section{Publisher's Note}

Springer Nature remains neutral with regard to jurisdictional claims in published maps and institutional affiliations.

\section{Submit your manuscript to a SpringerOpen ${ }^{\circ}$ journal and benefit from:}

- Convenient online submission

- Rigorous peer review

- Open access: articles freely available online

- High visibility within the field

- Retaining the copyright to your article

Submit your next manuscript at $\boldsymbol{\nabla}$ springeropen.com 